\title{
Candida Pneumonia: An Innocent Bystander or a Silent Killer?
}

\author{
Durga Shankar Meena Deepak Kumar \\ Department of Internal Medicine, All India Institute of Medical Sciences, Jodhpur, India
}

\section{Highlights of the Study}

- The existence of Candida pneumonia is debated due to its rarity and difficulty in diagnosis.

- Due to frequent Candida colonization in the respiratory tract, the decision to start antifungal therapy is always a challenge.

- Candida-bacterial synergy can potentiate the underlying disease; antifungal therapy in such cases may improve the outcome.

\section{Keywords}

Candida $\cdot$ Colonization · Pneumonia · Antifungal treatment · Beta-D-glucan

\begin{abstract}
Invasive candidiasis is predominantly seen in immunosuppressed patients and carries a significant mortality. The clinical spectrum of invasive candidiasis encompasses candidemia and disseminated infection (intra-abdominal abscess, osteomyelitis, endophthalmitis, and Candida meningitis). The existence of Candida pneumonia has been largely debated over the years due to its rarity and presence of frequent colonization. Demonstration of Candida species by lung biopsy along with evidence of inflammation is the only way to confirm this entity. The interpretation of Candida in respiratory specimens and the decision to initiate antifungal therapy is controversial due to the lack of clinical evidence. In this mini-review, we discuss the currently available clinical
\end{abstract}

karger@karger.com www.karger.com/mpp

Karger $\stackrel{\text { ' }}{5}$
(C) 2021 The Author(s)

Published by S. Karger AG, Basel

This is an Open Access article licensed under the Creative Commons Attribution-NonCommercial-4.0 International License (CC BY-NC) (http://www.karger.com/Services/OpenAccessLicense), applicable to the online version of the article only. Usage and distribution for commercial purposes requires written permission. data from the literature on Candida pneumonia and future perspectives regarding the need for antifungal therapy in such patients.

(c) 2021 The Author(s)

Published by S. Karger AG, Basel

\section{Introduction}

The incidence of fungal pneumonia is rising with the increasing use of immunosuppressants, inadvertent use of antimicrobials, newer diagnostic modalities, and increased survival of immunodeficient patients [1]. The common organisms that cause fungal pneumonia are Aspergillus, Cryptococcus, Mucor, and Pneumocystisjirovecii $[2,3]$. Despite encompassing more than two-thirds of cases of fungal infections, invasive candidiasis rarely manifests as Candida pneumonia. Invasive candidiasis is defined as the presence of Candida in the blood (candidemia) or deep-seated Candida infections such as intra- 
abdominal abscess, osteomyelitis, or renal abscess [4]. The presence of Candida isolated from respiratory specimens is usually regarded as colonization. The diagnosis of Candida pneumonia is difficult due to the nonspecific clinical and radiological features and the lack of specific biomarkers which necessitates the histopathological demonstration of the organism for confirmation of diagnosis. The rarity of Candida pneumonia makes the existence of this entity a matter of ongoing debate. In this review, we highlight the current evidence regarding the existence of Candida pneumonia and discuss the pros and cons of antifungal therapy.

\section{Epidemiology of Candida Pneumonia}

Candida is considered a part of the normal microflora of the skin, oral cavity, gastrointestinal mucosa, respiratory tract, and genitourinary tract [5]. There are currently no methods that can differentiate between commensalism, colonization, and infection with Candida species. In immunocompromised patients, Candida is frequently isolated from respiratory samples (sputum or bronchoalveolar lavage $[\mathrm{BAL}])$, though it should be interpreted with great caution. Clinical data regarding the diagnosis of Candida pneumonia are largely anecdotal, with a majority of studies based on autopsy findings. The true incidence of Candida pneumonia ranges from $0.23 \%$ to $0.4 \%$ $[6,7]$. However, these studies were conducted in highrisk groups (patients with hematological malignancies with severe neutropenia) which probably reflect selection bias. The true incidence may be much lesser than what is actually reported, which makes the existence of this entity even more controversial. A study by Schnabel et al. [8] highlighted this subject with an attempt to identify the incidence of Candida pneumonia in critically ill patients. They analyzed 701 BAL samples and described Candida as an etiology of pneumonia in only 5 patients $(0.7 \%)$. Out of these 5 patients, only 1 was immunocompetent. Another prospective study described the true incidence of Candida pneumonia in nonneutropenic critically ill patients, which was 8\% [9], although Candida isolation from the biopsy sample was $40 \%$ in this report. The existence of Candida pneumonia is also debated by Meersseman et al. [10] who did not find a single proven case in patients with the identification of Candida in histopathology. Despite the high incidence of Candida isolation $(40-56 \%)$ in respiratory samples (BAL and sputum) in all aforementioned studies, true Candida pneumonia remains a rare entity [8-10].

Candida Pneumonia: An Innocent

Bystander or a Silent Killer?

\section{Pathophysiology, Clinical Presentation, and Diagnosis}

Colonization of the respiratory epithelium by Candida species is a prerequisite of invasive infections. The predominant risk factors for invasive candidiasis are immunocompromised host, repeated use of broad-spectrum antibiotics, severe neutropenia, chemotherapy-induced mucositis, and prolonged hospital stay $[11,12]$. In addition, there are several other intrinsic factors like the release of hydrolytic enzymes and an ability to change from yeast form to hyphae which is a more virulent form that causes invasive disease. Hematogenous spread and microaspiration of oropharyngeal and gastric contents are the main triggers for Candida lung infiltration. The concurrent esophageal candidiasis and upper respiratory tract colonization were found commonly in patients with pulmonary candidiasis. The clinical presentation is diverse and nonspecific with many patients with profound neutropenia only having fever as a presenting symptom. Radiological findings are also nonspecific, which makes the diagnosis even more challenging. Bronchopneumonia, nodular opacities, lung abscess, and cavitary lesion are described as X-ray finding of cases [13-15]. In CTthorax, the most common finding is the presence of nodules. Cases with concomitant presence of Candida in bloodstream (candidemia) or evidence of disseminated candidiasis can be identified by isolation of the organism from blood or tissue sample. However, it is difficult to establish a causal association between Candida and respiratory illness even after successful isolation of Candida spp. from respiratory specimens. Currently, no culture or molecular-based diagnosis is available to differentiate between colonization and invasive candidiasis. Isolation of Candida from sputum samples should be discouraged, which always indicates contamination. The role of serological assay like beta-D-glucan (BDG) in invasive candidiasis was analyzed by Su et al. [16]. According to their report, endotracheal aspirate and BAL BDG were better predictors of suspected pulmonary candidiasis in comparison with serum BDG. Measuring serum BDG has no value in predicting pulmonary Candida infections, particularly in the absence of concurrent candidemia. However, BDG can be positive in other fungal infections too (Aspergillus and $P$. jirovecii) which could be a limiting factor in confirming the diagnosis. Thus, BAL BDG positivity without isolation of Candida in a respiratory specimen does not have much significance. We advocate the combined use of BAL BDG and isolation of Candida from respiratory samples (BAL/tracheal aspirate) for the diagnosis of Candida pneumonia particularly in conditions in

Med Princ Pract 2022;31:98-102

DOI: $10.1159 / 000520111$ 
which histopathological examination is not feasible. In addition, the aforementioned approach should be rewarding in cases with a high pretest probability of pulmonary candidiasis. Further prospective studies on a large scale are needed to validate this approach. The germ tube antigen, mannan antigen/antibody detection, and PCR methods have not been validated for the detection of Candida pneumonia. The only method to confirm the diagnosis is obtaining a lung biopsy and demonstration of yeast or hyphae along with inflammatory cells, which is sometimes not viable in critically ill patients.

\section{When Should Candida Pneumonia Be Treated?}

Treatment of Candida pneumonia is largely debated with the lack of practice guidelines due to the rarity of disease and difficulty in diagnosis. Interpretation among clinicians on the isolation of Candida species is variable, which can be contamination, commensalism, colonization, or invasive disease. So, the decision regarding antifungal therapy in such patients remains controversial. Rising drug resistance (especially in the cases of Candida krusei and Candida auris), adverse effects, and treatment cost are the major concerns in cases with inadvertent antifungal therapy.

In cases with biopsy-proven candidiasis, the decision is straightforward. Echinocandins are the drug of choice for invasive candidiasis [4]. However, the poor clinical condition of the patients may preclude histological diagnosis in most cases. The initiation of therapy based on BAL positivity is not recommended due to the poor correlation of BAL findings with biopsy [17]. A study by Wood et al. [17] described the positive and negative predictive value of BAL culture, $29 \%$ and $89 \%$, respectively. Furthermore, in their report, only $2 \%$ of patients were given antifungal therapy, and none of the patients developed candidemia despite not receiving antifungal treatment. The mortality rate was also found similar in patients who did not receive antifungal therapy in comparison with those who received treatment based on isolation of BAL Candida [17]. Another report by Terraneo et al. [18] described that isolation of Candida from the respiratory sample may increase the initial disease severity, although the overall outcome did not change regardless of antifungal treatment. A similar observation was reported by Lindau et al. [19] who concluded that the treatment of Candida pneumonia did not improve the outcome. Another interesting theory was to hide the identification of Candida species in a respiratory specimen and its impact on the outcome of patients with clinically suspected pneumonia. Barenfanger et al. [20] reported the benefits of limiting the identification of Candida; by using this approach, they have shown significant reduction in mortality, cost, length of hospital stay, and adverse events related to antifungal therapy. Therefore, initiation of antifungal in immunocompetent patients is not warranted. However, the use of antifungal drugs in immunocompromised patients with presence of respiratory Candida is less clear. Whether the presence of Candida in such patients should be ignored remains questionable.

There are reports describing the interaction of Candida species and various bacterial organisms (particularly Pseudomonas and Staphylococcus species) which are common in the setting of ICU (ventilator-associated pneumonia) [21-23]. The detection of Candida in the setting of ventilator-associated pneumonia (VAP) was reported as an independent predictor of increases in mortality and length of hospital stay $[21,22]$. A recent report by Moss and Musher [24] highlighted the contributory role of Candida species in community-acquired pneumonia (CAP). In 6 out of 154 consecutive CAP patients, Candida was isolated from high-quality sputum. All patients had bacterial coinfections (Staphylococcus aureus, S. pneumoniae, and Pseudomonas aeruginosa). Three patients had raised BDG levels, and 1 patient had candidemia. However, clinical improvement could not be attributed to Candida because all patients were given antifungals and antibiotics [24]. Roux et al. [21] reported the synergistic effect of Candida albicans and Pseudomonas in patients with pneumonia, and those with Candida colonization were more likely to have a VAP due to Pseudomonas. A majority of the available data are retrospective and based on animal models, which showed increased virulence of Pseudomonas, $S$. aureus, and E. coli in the presence of Candida colonization. The validity of these findings was debated by critics who suggested that the presence of Candida in the respiratory tract is just an indicator of lymphocyte dysfunction and severe immunodeficiency $[19,25]$. Candida albicans can also promote the mixed-species biofilm formation (along with S. aureus) which confers more antimicrobial resistance and persistence of pathogenic bacteria inside the respiratory tract [26]. Yu et al. [27] reported the effect of antifungal therapy in decreasing the levels of inflammatory markers (interleukin-6, tumor necrosis factor- $\alpha$, and interferon- $\gamma$ ) in hospital-acquired bacterial pneumonia (Acinetobacter baumannii and P. aeruginosa) with Candida colonization. Furthermore, they reported a significant increase in $\mathrm{CD} 4^{+}$lymphocytes after antifungal therapy [27]. Conversely, a report by Ader et al. [28] described 
Table 1. Candida species-bacterial interaction and its impact on the outcome of respiratory infections

1. Increased adhesion of Candida to the respiratory epithelium in the presence of bacteria

2. Formation of mixed-species biofilm (e.g., Candida albicans with Pseudomonas aeruginosa and Staphylococcus aureus)

3. Delayed clearance of GNB by augmenting the antibiotic resistance in the presence of Candida colonization

4. Promotes the switching from yeast to hyphae form and increase invasion of the respiratory epithelium

5. Candida albicans enhances the virulence of GNB with the help of quorum-sensing molecules like farnesol

6. Inflammatory response (increased interleukins and interferon gamma) produced by Candida colonization which makes GNB less susceptible to phagocytosis by alveolar macrophages

GNB, Gram-negative bacteria.

the decreased epithelial permeability, bacterial load ( $P s e u-$ domonas spp.) and bronchiole inflammation in mice with pre-existing Candida albicans colonization. Interestingly, this effect was reversed when an antifungal drug (caspofungin) was added [28]. The evidence from the literature regarding the cross-kingdom interaction of Candida and various bacteria is summarized in Table 1 . The only randomized study was published in 2014 (CANTREAT TRI$\mathrm{AL}$ ) which did not find any role of antifungal drugs in reducing the inflammatory markers and improving the innate immunity in patients with VAP with Candida colonization [29].

\section{Conclusion}

Due to the lack of in vivo data and prospective and randomized studies, the findings described above cannot be extrapolated to all immunocompromised patients with isolation of Candida. The decision to start antifungal treatment should be done on a case-by-case basis. Antifungal therapy may be given to those who are immunodeficient, not improving with antibiotics, with positive biomarkers (BDG) and without an alternative etiology. Despite the abundance of data from animal models, further clinical evidence is needed; until then, antifungal treatment in Candida pneumonia remains a matter of debate.

\section{Conflict of Interest Statement}

The authors declare that they have no conflicting interests.

\section{Funding Sources}

This review article did not receive any internal or external funding.

\section{Author Contributions}

D.S.M. contributed to concept and design of the study; D.S.M. and D.K. contributed to analysis of the data and drafting of the manuscript. Both authors critically revised the manuscript, approved the final version to be published, and agree to be accountable for all aspects of the work.

\section{References}

1 Lamoth F, Alexander BD. Nonmolecular methods for the diagnosis of respiratory fungal infections. Clin Lab Med. 2014;34:315-36.

2 Nucci M, Marr KA. Emerging fungal diseases. Clin Infect Dis. 2005;41:521-6.

3 Park BJ, Pappas PG, Wannemuehler KA, Alexander BD, Anaissie EJ, Andes DR, et al. Invasive non-Aspergillus mold infections in transplant recipients, United States, 20012006. Emerg Infect Dis. 2011;17:1855-64.

4 Pappas PG, Lionakis MS, Arendrup MC, Ostrosky-Zeichner L, Kullberg BJ. Invasive candidiasis. Nat Rev Dis Primers. 2018;4: 18026.

5 Kumamoto CA, Vinces MD. Alternative Candida albicans lifestyles: growth on surfaces. Annu Rev Microbiol. 2005;59:113-33.
6 Masur H, Rosen PP, Armstrong D. Pulmonary disease caused by Candida species. Am J Med. 1977 Dec;63(6):914-25.

7 Haron E, Vartivarian S, Anaissie E, Dekmezian R, Bodey GP. Primary Candida pneumonia. Experience at a large cancer center and review of the literature. Medicine. 1993;72: $137-42$.

8 Schnabel RM, Linssen CF, Guion N, van Mook WN, Bergmans DC. Candida pneumonia in intensive care unit? Open Forum Infect Dis. 2014; 1:ofu026.

9 el-Ebiary M, Torres A, Fàbregas N, de la Bellacasa JP, González J, Ramirez J, et al. Significance of the isolation of Candida species from respiratory samples in critically ill, non-neutropenic patients. An immediate postmortem histologic study. Am J Respir Crit Care Med. 1997;156:583-90.

10 Meersseman W, Lagrou K, Spriet I, Maertens J, Verbeken E, Peetermans WE, et al. Significance of the isolation of Candida species from airway samples in critically ill patients: a prospective, autopsy study. Intensive Care Med. 2009;35:1526-31.

11 Krause R, Halwachs B, Thallinger GG, Klymiuk I, Gorkiewicz G, Hoenigl M, et al. Characterisation of Candida within the mycobiome/ microbiome of the lower respiratory tract of ICU patients. PLoS One. 2016;11:e0155033.

12 Rollenhagen C, Mamtani S, Ma D, Dixit R, Eszterhas S, Lee SA. The role of secretory pathways in Candida albicans pathogenesis. J Fungi. 2020;6:26.
Candida Pneumonia: An Innocent

Bystander or a Silent Killer?
Med Princ Pract 2022;31:98-102 
13 Dermawan JKT, Ghosh S, Keating MK, Gopalakrishna KV, Mukhopadhyay S. Candida pneumonia with severe clinical course, recovery with antifungal therapy and unusual pathologic findings: a case report. Medicine. 2018;97:e9650.

14 Petty LA, Gallan AJ, Detrick JA, Ridgway JP, Mueller J, Pisano J. Candida dubliniensis pneumonia: a case report and review of literature. Mycopathologia. 2016;181:765-8.

15 Tan M, Wang J, Hu P, Wang B, Xu W, Chen J. Severe pneumonia due to infection with Candida krusei in a case of suspected Middle East respiratory syndrome: a case report and literature review. Exp Ther Med. 2016;12: 4085-8.

$16 \mathrm{Su}$ KC, Chou KT, Hsiao YH, Tseng CM, Su VY, Lee YC, et al. Measuring (1,3)- $\beta$-Dglucan in tracheal aspirate, bronchoalveolar lavage fluid, and serum for detection of suspected Candida pneumonia in immunocompromised and critically ill patients: a prospective observational study. BMC Infect Dis. 2017; 17:252.

17 Wood GC, Mueller EW, Croce MA, Boucher BA, Fabian TC. Candida sp. isolated from bronchoalveolar lavage: clinical significance in critically ill trauma patients. Intensive Care Med. 2006;32:599-603.
18 Terraneo S, Ferrer M, Martín-Loeches I, Esperatti M, Di Pasquale M, Giunta V, et al. Impact of Candida spp. isolation in the respiratory tract in patients with intensive care unitacquired pneumonia. Clin Microbiol Infect. 2016;22:94.e1-e8.

19 Lindau S, Nadermann M, Ackermann H, Bingold TM, Stephan C, Kempf VA, et al. Antifungal therapy in patients with pulmonary Candida spp. colonization may have no beneficial effects. J Intensive Care. 2015;3:31.

20 Barenfanger J, Arakere P, Cruz RD, Imran A, Drake C, Lawhorn J, et al. Improved outcomes associated with limiting identification of Candida spp. in respiratory secretions. Clin Microbiol. 2003;41:5645-9.

21 Roux D, Gaudry S, Khoy-Ear L, Aloulou M, Phillips-Houlbracq M, Bex J, et al. Airway fungal colonization compromises the immune system allowing bacterial pneumonia to prevail. Crit Care Med. 2013;41:e191-9.

22 Delisle MS, Williamson DR, Albert M, Perreault MM, Jiang X, Day AG, et al. Impact of Candida species on clinical outcomes in patients with suspected ventilator-associated pneumonia. Can Respir J. 2011;18:131-6.

23 Tan X, Chen R, Zhu S, Wang H, Yan D, Zhang $\mathrm{X}$, et al. Candida albicans airway colonization facilitates subsequent Acinetobacter baumannii pneumonia in a rat model. Antimicrob Agents Chemother. 2016;60:3348-54.
24 Moss BJ, Musher DM. Candida species in community-acquired pneumonia in patients with chronic aspiration. Pneumonia. 2021; 13(1):12.

25 Pendleton KM, Huffnagle GB, Dickson RP. The significance of Candida in the human respiratory tract: our evolving understanding. Pathog Dis. 2017;75:ftx029.

26 Morales DK, Hogan DA. Candida albicans interactions with bacteria in the context of human health and disease. PLoS Pathog. 2010;6: e1000886.

27 Yu Y, Li J, Wang S, Gao Y, Shen H, Lu L. Effect of Candida albicans bronchial colonization on hospital-acquired bacterial pneumonia in patients with systemic lupus erythematosus. Ann Transl Med. 2019;7:673.

28 Ader F, Jawhara S, Nseir S, Kipnis E, Faure K, Vuotto F, et al. Short term Candida albicans colonization reduces Pseudomonas aeruginosa-related lung injury and bacterial burden in a murine model. Crit Care. 2011;15:R150.

29 Albert M, Williamson D, Muscedere J, Lauzier F, Rotstein C, Kanji S, et al. Candida in the respiratory tract secretions of critically ill patients and the impact of antifungal treatment: a randomized placebo controlled pilot trial (CANTREAT study). Intensive Care Med. 2014;40:1313-22. 